\title{
Social facilitation and sensory deprivation in operant behavior of rats
}

VLADIMIR PISHKIN AND JAY T, SHURLEY 1

VA HOSPITAL AND UNIVERSITY OF OKLAHOMA SCHOOL OF MEDICINE

Female albino rats $(\mathrm{N}=35)$ were used in a study designed to test the effects of social and solitary conditions 6 months before and during $40 \mathrm{hr}$. of sensory deprivation (SD). Significant social facilitation of operant behavior rates subsequent to SD was demonstrated. Re sults were compared to earlier findings; influences of illumination and the 6 month, pre-SD period were discussed.

In a recent paper on "social facilitation" in humans and animals Zajonc (1965) reviewed a number of studies with conflicting results and attempted some tentative explanations for the inconsistent findings. In short, it is not clear under what circumstances social facilitation occurs and what influence it may have on learning and stress. Moyer \& Korn (1965), for instance, have shown that isolated albino rats are more emotional than group-raised Ss. Open field tests have also revealed that isolated Ss are more emotional than group-housed rats (Hahn, 1965). In an earlier experiment Hatch et al (1963) concluded that individual isolation of rats, for 3 months, was stressful and resulted in ascending caudal dermatitis in $100 \%$ of the isolated rats and that they were "aggressive and intractable animals." On the other hand, there is evidence from some time ago that solitary cockroaches needed significantly less time (Gates \& Allee, 1933) to learn an E-shaped maze than grouped Ss. Thus, although evidence is clear that there is social facilitation in human problem solving from earlier (Allport, 1924) and more recent findings (Pishkin \& Blanchard, 1963), this evidence for facilitation in learning is not always consistent with animal results.

Since in a previous study with individual rats (Pishkin \& Shurley, 1966) it was shown that sensory deprivation (SD) was stressful and profoundly diminished performance on an operant task, the present study was designed to test the effects of social isolation and dyadic group living before and during SD upon subsequent operant conditioning. It was hypothesized that negative, stress effects of SD would be diminished in social conditions, where presence of another animal would be an additional source of sensory input and that rats caged in pairs would show superior performance on a subsequent operant task.

\section{Method}

The Ss were 35 female albino rats of the Holtzman strain with an average weight of $264 \mathrm{gm}$. They were 52 days old at the start of the experiment. The first phase of this study consisted of splitting the Ss into two groups: 10 rats, at random, were individually caged (IC) and the remaining 20 were randomly paired and caged (GC) in groups of two. The wire mesh cages measured $10 \times 8 \times 7 \mathrm{in}$. and the rats were maintained on an ad libitum diet of water and food (Rockland Mouse/ Rat Diet) for 6 months before SD, at which time they were placed into a SD chamber either in pairs or alone. Half of the IC rats were exposed to SD individually (ISD), the other half were paired in groups of two with strange rats in the deprivation chamber (GSD). The GC sample was likewise split in half; five rats were placed in SD individually (ISD), the other five remained in groups of two with their partner throughout the SD period (GSD).

Thus, the four groups are represented as follows: IC-ISD, IC-GSD, GC-ISD and GC-GSD; the first two letters refer to the 6 month pre-SD period followed by individual or group SD. All rats, in pairs or alone, were exposed to the isolation chamber for $24 \mathrm{hr}$. of familiarization and were on ad lib diet with no reduction of visual nor auditory inputs. Familiarization period was followed by $40 \mathrm{hr}$, of SD and water deprivation. When the $40 \mathrm{hr}$. SD period was completed all rats were individually placed into a Skinner box with a water reward dipper (capacity .02 cc) and a conditioning bar. The SD chamber was an Industrial Acoustics Co. Mod. AC-1 (sound absorption coefficient of .99 at a frequency of $1000 \mathrm{cps}$ ) and the Stoelting Skinner Box were the same as utilized in the previous study (Pishkin \& Shurley, 1966). The chamber was equipped with a quiet ventilation fan and was totally dark throughout the $40 \mathrm{hr}$. period; chamber temperature was kept constant at $72^{\circ} \mathrm{F}$. Results and Discussion

Cumulative bar-pressing responses by the four groups of rats are represented in Fig. 1. Clearly, it is shown that IC-GSD rats had the highest rate of response while the GC-GSD Ss were second in number of emitted bar-presses. The marked difference between the rats who were paired in SD (GC-GSD and IC-GSD) and the other two groups, who were individually isolated, is striking. The differences between IC-GSD and IC-ISD $(\mathrm{U}=2, \mathrm{p}<.02), \mathrm{IC}-\mathrm{GSD}$ and GC-ISD $(\mathrm{U}=2, \mathrm{p}<.02)$, GC-GSD and GC-ISD $(U=3, p<.03), G C-G S D$ and IC-ISD $(U=4, p<.05)$ were all significant; none of the other differences were reliable. It is noteworthy that superior performance was found in the rats who were individually caged, but were then paired with another rat during the SD period.

Although conditioning rate of the group-caged rats (GSD) was not significantly different statistically from that of the individually caged $\mathrm{Ss}$, it is suggested that introduction of another animal, following individual housing for 6 months, resulted in a change to a more novel situation, with relative increased sensory input 


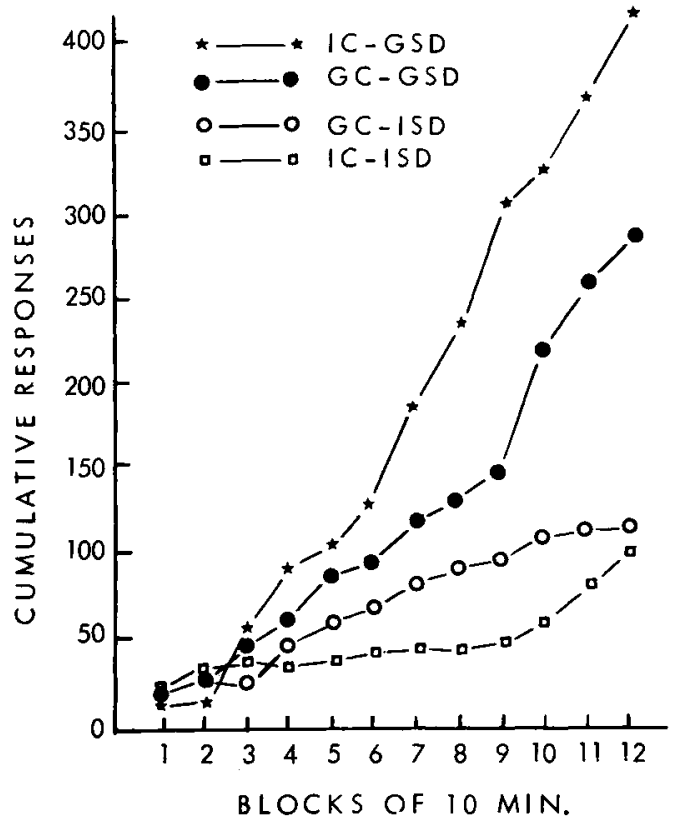

Fig. 1. Cumulative bar-press responses over the 2 -hr. period. Each point represents total responses by 5 rats. The letters represent: individually caged-groups in SD (IC-GSD), group cagedgroups in SD (GC-GSD), group caged-individuals in SD (GC-ISD) and individually caged-individuals in SD (IC-ISD).

as compared to the GC-GSD sequence of events. Accordingly, the stressful, negative effects of SD were not pronounced to the same degree in the condition where "social input" was newly introduced. It is speculated that the increase of novel social arousal partially compensated for the reduction of stimulus impingement during SD

Interestingly, the performances of all groups were inferior to those of the previous study (Pishkin \& Shurley, 1966) wherein the chamber was illuminated during the SD period. Since the present GC-ISD condition replicates a $40-\mathrm{hr}$. SD condition of the earlier study in every respect, except that in the earlier experiment the SD chamber was illuminated, direct comparisons of the matching groups' performances was made in order to assess the effects of illumination in SD. The medians of bar-presses were 93 and 27, respectively, by rats in the original, Pishkin \& Shurley (1966) study (illuminated chamber, GC-ISD), and the present sample (GC-ISD). Moreover, the difference between these two groups was significant $(U=6, p<.05)$, manifesting stress-potentiating effects of darkness plus quiet in SD, which were reflected by noticeably reduced bar-press rate in subsequent performance. Even the superior group of the present experiment(IC-GSD) produced a total of only 428 cumulative responses (five rats) in $2 \mathrm{hr}$. as compared to 2357 bar-presses $(U=2$, $\mathrm{p}<.01$ ) by seven control rats of the earlier study who were not exposed to SD (Pishkin \& Shurley, 1966).

There is one subtle aspect of the present results that needs some consideration. The fact that rats caged in solitude for 6 months performed at approximately the same level as the rats who were paired, also for 6 months (individual SD in both groups), reveals that emission of operant response is not socially facilitated during the pre-SD period. On the other hand, it may be speculated that solitary SD is sufficiently profound to extinguish any differential stress effects of social isolation upon subsequent conditioning. Therefore, the effects may have become obscured as a function of either isolated or paired pre-SD housing for a 6 month period (see Fig. 1). This question can be answered only by introducing operant conditioning at the end of the 6 month period antecedent to SD.

While the facilitating effect upon "learning," as measured by operant behavior, is clearly evident in the superior performance of the rats exposed to SD in familiar or unfamiliar pairs over those exposed individually, the more striking finding is that "learning" is inhibited to a greater degree by the experimental condition of quiet plus darkness as the SD condition, than it is facilitated by the circumstance of having a fellow rat to share the ordeal with him. Since the rat is normally a nocturnal animal, the deleterious effect of darkness added to quiet upon subsequent learning is somewhat of a surprise. Could it be that the effect of denying the animal information through the two modalities of sight and hearing at once is more than twice as disrupting as denying him either one alone?

Shurley (1962), in human sensory isolation experiments, concluded that, for one human, the most potent source of sensory stimuli is another human, preferably of the opposite sex, so that "group" exposure to the SD environment to some extent vitiates the experimental aim of SD. This would seem to be true for the rat species as well, although the question of the influence of sex differences was not considered in this experiment.

\section{References}

Allport, F. H. Social psychology. Boston: Houghton-Mifflin, 1924. Gates, M. S., \& Allee, w. C. Conditioned behavior of isolated and grouped cockroaches on a simple maze. J. comp. Psychol., 1933, 15,331 .

Hahn, W. W. Some effects of group size on behavior and physiology of the rat. J. psychosom. Res., 1965, 8, 455-465.

Hatch, A., Wiberg, G. S., Balazs, T., \& Grice, H. C. Long-term isolation stress in rats. Science, 1963, 142, 507.

Moyer, K. E., \& Korn, J. H. Behavioral effects of isolation in the rat. Psychon. Sci., 1965, 3, 503-504.

Pishkin, V., \& Blanchard, R. J. Stimulus and social cues in concept identification of schizophrenics and normals. J. abnorm. soc. Psychol., 1963, 67, 454-463.

Pishkin, V., \& Shurley, J. T. Sensory deprivation and operant conditioning of rats. Psychon. Sci., 1966, 5, 283-284.

Shurley, J. T. Problems and methods in experimental sensory input alteration and invariance. In Tourlentes, T. T. (Ed.), Research approaches to psychiatric problems. New York: Grune \& Stratton, 1962. Pp. 145-160.

Zajonc, R. B. Social facilitation. Science, 1965, 149, 269-274. Note

1. The authors are indebted to Linn $W$. Wainner and Lewis $M$. Jones for their assistance. This project was supported by VA Medical Research Funds. 